\title{
Cell Therapy for Kidney Injury: Different Options and Mechanisms - Kidney Progenitor Cells
}

\author{
Kenji Osafune \\ Center for iPS Cell Research and Application (CiRA), Kyoto University, Kyoto, Japan
}

\section{Key Words}

Cell therapy · Acute kidney injury · Kidney progenitor cells

\begin{abstract}
Background: Since no specific or radical treatments have yet been established for acute kidney injury (AKI), the development of cell transplantation therapy using renal progenitors is desirable as a new therapeutic option for AKI. The recent advances in developmental biology, stem cell biology, and nephrology have led to an increased availability of renal progenitors from multiple sources. Summary: Four main sources of renal progenitors have been described so far: isolation from (1) embryonic or (2) adult kidneys, (3) directed differentiation of pluripotent stem cells such as embryonic stem cells (ESCs) and induced pluripotent stem cells (iPSCs), and (4) cellular reprogramming of fully differentiated adult renal cells. Renal progenitors from adult kidneys may not be equivalent to those from embryonic kidneys, and they contain several different cell populations identified by various methods. The methods used for the directed differentiation of ESCs/iPSCs and reprogramming of differentiated adult renal cells into renal progenitors have not been fully established. The therapeutic effects of progenitor cell transplantation in AKI animal
\end{abstract}

models have been examined in a small number of reports using renal progenitors from adult kidneys, while no reports have described the therapeutic potential of renal progenitors from other sources. Key Messages: Renal progenitor transplantation might provide a novel therapeutic strategy for AKI. Further research efforts toward the clinical application of this strategy are needed, including a detailed characterization of embryonic or adult renal progenitors and the development of in vitro expansion methods and therapeutically effective transplantation methods for these cell types. More experience and knowledge should be accumulated regarding the directed differentiation of pluripotent stem cells and cellular reprogramming to generate renal progenitor cells.

(c) 2014 S. Karger AG, Basel

\section{Introduction}

Acute kidney injury (AKI) is a common clinical entity in hospitalized patients, and it is associated with high morbidity and mortality rates, particularly in those with advanced age, diabetes, chronic kidney disease, and vascular diseases [1]. The current treatments for AKI are

\section{KARGER}

E-Mail karger@karger.com www.karger.com/nee (c) 2014 S. Karger AG, Basel

1660-2129/14/1262-0064\$39.50/0
Kenji Osafune

53 Kawahara-cho, Shogoin, Sakyo-ku

Kyoto 606-8507 (Japan)

E-Mail osafu@cira.kyoto-u.ac.jp 
mainly supportive, and there are no definitive therapeutic modalities currently available for these patients. Among the candidate strategies to treat AKI is the development of cell transplantation therapy using progenitor cells.

Stem cells are defined as cells that have self-renewal capacity and can give rise to terminally differentiated cells constituting their original tissues. Progenitor cells, which differ from stem cells, are more developmentally committed to some cell lineages than stem cells, but they are nevertheless undifferentiated or immature in biological nature compared to fully differentiated and mature cells. It is sometimes difficult to clearly distinguish progenitor cells from stem cells, and both cell types are often equated. Therefore, the two cell types specific for kidneys will be described as one entity, i.e. 'renal stem/progenitor cells' or 'renal progenitors', in this review.

The tissue-specific stem/progenitor cells of other organs have also been investigated for their therapeutic effects in AKI treatments, including mesenchymal stem cells of various origins [2]. These approaches are described in other reviews in this issue.

In contrast to tissue-specific stem cells, pluripotent stem cells, such as embryonic stem cells (ESCs) [3, 4] and induced pluripotent stem cells (iPSCs) [5], have a virtually unlimited replicative capacity in culture dishes and are theoretically able to give rise to any cell type in the body. These stem cells have been increasingly used as sources for cell replacement therapy. Like the generation of other clinically useful cell types, vigorous efforts to differentiate renal lineage cells have already been made, mainly using mouse ESCs, while the methods needed to induce human ESCs or iPSCs into renal lineage cells have not yet been fully established [6].

Recently, there has been an increasing number of reports describing a successful generation of the desired cell types via manipulation of the core transcriptional factors, converting from fully differentiated cells to a pluripotent state or from one fully differentiated cell type into another [7]. This experimental strategy is called cellular reprogramming, and it has already been utilized to generate renal progenitors [8].

This review will summarize the current status of research on the identification or isolation of renal stem/ progenitor cells from embryonic or adult kidneys, the directed differentiation of pluripotent stem cells into renal progenitors, and cellular reprogramming to generate renal progenitors from other cell types. The therapeutic potential of progenitor cell transplantation for treating AKI will be also described.

Treatment of AKI Using Progenitor Cells

\section{Stem/Progenitor Cells in the Embryonic Kidney}

The kidneys are derived from an early embryonic germ layer, the intermediate mesoderm (IM), which is located between the lateral and paraxial mesoderms [9]. In vertebrates, the IM successively develops 3 types of kidneys: the pronephros, the mesonephros, and the metanephros. The 3 kidneys are similar in that they consist of a basic functional unit, i.e. the nephron, although the number of nephrons differs among the kidneys. The mammalian adult kidney, i.e. the metanephros, is formed by reciprocal interactions between 2 tissues derived from IM: the metanephric mesenchyme (MM) and the ureteric bud. The ureteric bud induces the MM to differentiate into epithelialized nephrons and interstitium, while the MM directs the ureteric bud to elaborate the lower urinary tract, from the collecting ducts through the renal pelvis and ureter to part of the urinary bladder.

By creating a clonogenic assay system which uses stimulation of Wnt4, an epithelializing factor for MM in embryonic development, we previously demonstrated that the MM contains multipotent nephron progenitors that can give rise to several kinds of epithelial cells, such as glomerular podocytes and epithelia of the proximal and distal renal tubules and Henle's loop [10]. The progenitor cells are contained only in the cell population strongly expressing Sall1, a zinc finger transcription factor that is essential for kidney development, and can reconstitute 3 -dimensional kidney structures in an organ culture setting containing glomeruli- and tubule-like components. The presence of nephron progenitors was also demonstrated in the portion of the MM closest to the ureteric tips, i.e. the cap mesenchyme, using in vivo lineage-tracing experiments for Six2, an essential transcription factor for kidney development [11]. Self-renewing progenitors have also been isolated from embryonic mouse MM as nephrospheres by growing cells in a suspension culture, although they have displayed a limited nephrogenic capacity [12].

These embryonic nephron progenitors disappear by the time kidney development is completed, and they do not exist in adult mice even after AKI in ischemia-reperfusion models, which was elegantly demonstrated by a lineage-tracing study for Six2 [13]. In contrast, nephron progenitors were reported to still exist in the adult kidneys of zebrafish and to contribute to the de novo regeneration of nephrons after injury [14]. It was recently demonstrated that NCAM+ nephron progenitors from human embryos engrafted and integrated into diseased murine kidneys after transplantation, and that they

Nephron Exp Nephrol 2014;126:64-69 
Fig. 1. Sources of renal stem/progenitor cells. Renal progenitors are identified and isolated from embryonic (A) or adult kidneys (B). Directed differentiation using growth factors and small molecules can induce ESCs/iPSCs into renal progenitors by mimicking normal development (C). Reprogramming of fully differentiated renal cells, such as renal tubular cells, can be applied to generate renal progenitors in vitro using gene overexpression strategies (D). Note that embryonic and adult renal progenitors are not currently considered to be equivalent and that the directed differentiation of ESCs/iPSCs and cellular reprogramming experiments are mostly aimed at generating embryonic renal progenitors.

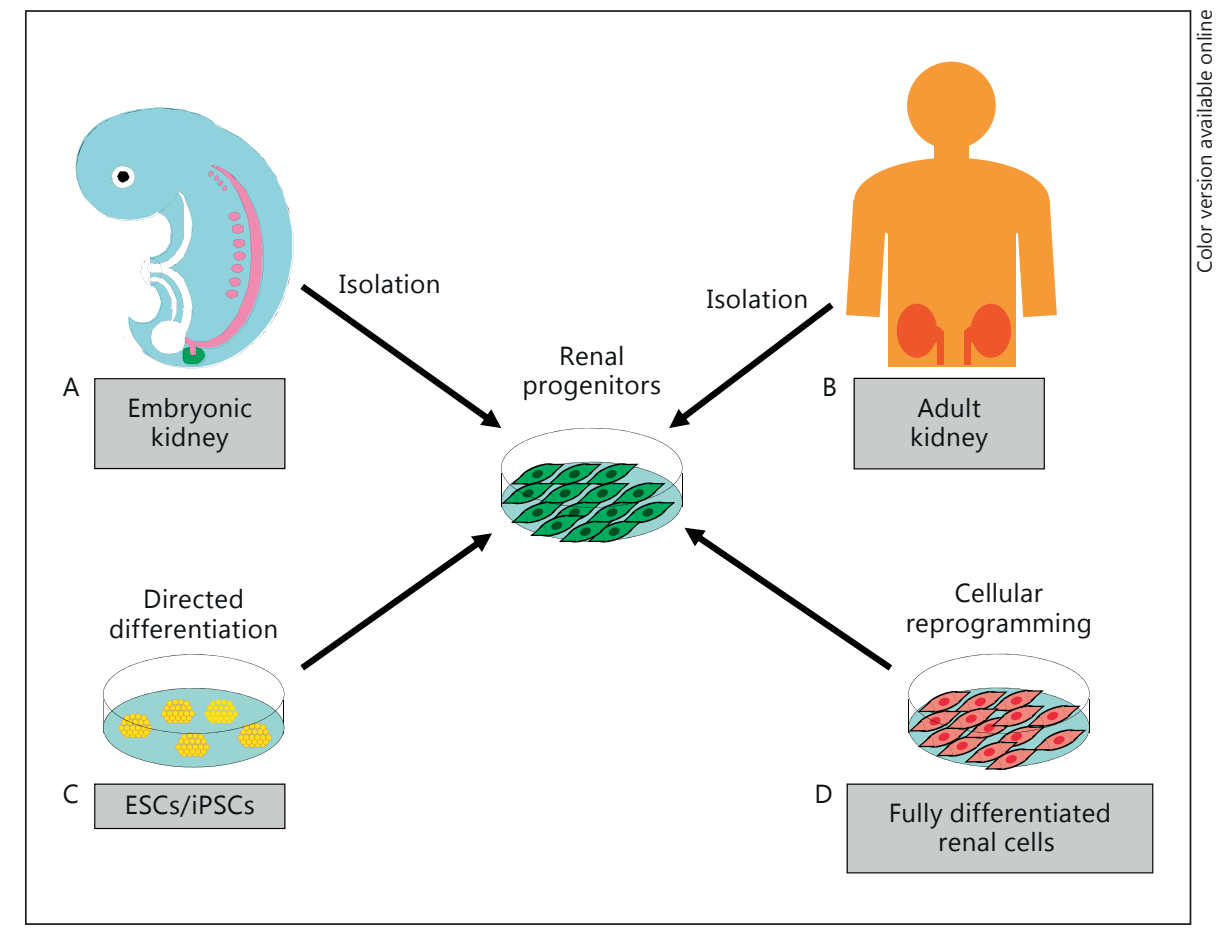

showed beneficial effects on chronic renal failure by halting disease progression in the 5/6 nephrectomy model [15]. However, no reports have examined the therapeutic potential of embryonic nephron progenitors in AKI models.

The detailed mechanisms regulating progenitor cell proliferation depending on their surrounding environment in embryonic kidneys, i.e. the niche, remain to be elucidated. Therefore, the in vitro expansion methods necessary for embryonic renal progenitors have not been established, and accelerated research towards the clinical application of transplantation therapy is needed.

\section{Stem/Progenitor Cells in the Adult Kidney}

Renal stem/progenitor cells have been reported to exist or have been isolated from various locations in adult kidneys, including the renal papilla [16], tubular epithelial cells $[17,18]$, Bowman's capsule [19-21], and the juxtaglomerular apparatus [22], via various identification methods such as label-retaining assays [16, 17], clonogenic assays [18], flow cytometry using cell surface markers [19-21], lineage tracing [22], and side population (SP) assays $[23,24]$. Although these adult progenitors contribute to the epithelia of glomeruli and renal tubules like embryonic progenitors, they may represent cell populations different from embryonic nephron progenitors, with different gene expression profiles and cellular origins.

Label-retaining cells (LRCs) examined by in vivo bromodeoxyuridine labeling exhibit the characteristic slow cycling of tissue-specific stem cells. One report showed that LRCs were found in the renal epithelial tubular cells of rat kidneys and that they contributed to the tubular regeneration after renal ischemia [17]. In contrast, another report identified LRCs in the interstitium or upper papilla, forming a compartment of rapidly proliferating cells that may have a role after acute injury [16].

One group generated clonally expanded cells from the S3 segment of rat proximal renal tubules, which are known to be most susceptible to injury and appear to have the highest proliferation rate in the nephron segments, showing a feature of stem cells. Transplantation of these progenitors significantly improved renal function in both cisplatin-induced and ischemia-reperfusion AKI models [18].

Regarding isolation using cell surface markers, a stem cell marker (Sca1) was used, and nontubular Sca1+ progenitor cells in the papillary interstitial space of the adult mouse kidney, which displayed some features overlapping those of the papillary LRCs described above, were 
identified [25]. It has been reported that a population of CD133+CD24+ cells localized at the urinary pole of Bowman's capsule in adult human, mouse, or rat kidneys represents renal progenitors that give rise to both glomerular podocytes and renal tubular epithelia. Clonally expanded $\mathrm{CD} 133+\mathrm{CD} 24+$ cells also contribute to the regeneration of tubular structures and significantly ameliorate the functional kidney damage after their intravenous injection into severe combined immunodeficiency mice with AKI induced by rhabdomyolysis [19, 20]. It has also been reported that these progenitor cells compose the hyperplastic glomerular lesion in several human proliferative glomerulonephritis [21].

The results of lineage-tracing experiments have demonstrated that cells of the renin lineage in the juxtaglomerular compartment serve as progenitors of glomerular podocytes and parietal epithelial cells in experimental glomerular disease [22].

SP cells are defined as a cell population that can efflux the DNA-binding dye Hoechst 33342 and may exhibit stem cell-like characteristics. SP cells in the bone marrow overlap hematopoietic stem cells, and the cell type has also been isolated from other tissues, including adult kidney, to examine their characteristics as tissue-specific stem cells. However, the injection of adult kidney SP cells did not show significant tubular integration in adriamycin- and cisplatin-induced nephropathy models, although the treatment reduced the renal damage. The result suggested that humoral factors from SP cells may be important for the amelioration of renal injury [23, 24].

Various types of adult renal progenitors identified by different methods have been reported. A detailed characterization of these cells and examination of the therapeutic potential of each progenitor cell population are required.

\section{Renal Progenitors Differentiated from Pluripotent Stem Cells or Reprogrammed from Adult Renal Cells}

ESCs are pluripotent stem cells derived from the inner cell mass of fertilized eggs in mammals, while iPSCs are generated from somatic cells via the transduction of reprogramming factors, such as the combination of Oct $3 / 4$, Sox2, Klf4, and c-Myc, and closely resemble ESCs [3-5]. After the successful derivation of human ESCs, regenerative medicine strategies have been considered with an eye toward clinical applications aimed at the functional recovery of dysfunctional organs via the transplantation of organ-specific cell types generated from ESCs in vitro.

Treatment of AKI Using Progenitor Cells
Moreover, iPSCs can overcome two issues associated with the use of human ESCs: the ethical problems related to the use of human fertilized eggs and the potential for immune rejection after transplantation due to antigenic differences between the ESCs and the patients. Thus, research on regenerative medicine using ESCs/iPSCs with a view towards clinical applications has gained increasing attention since the derivation of iPSCs.

Fundamental research aimed at establishing methods to efficiently induce mouse ESCs into renal progenitors and fully differentiated renal cells has already been carried out $[6,26]$. Some investigations used key transcriptional factors identified by studies on kidney development as markers for differentiation, such as Osr1 for the IM and Sall1 and Six2 for nephron progenitors in the MM. Most reports used combination treatments with nephrogenic growth factors or chemicals, such as activin A, bone morphogenetic protein 4 or 7 , and retinoic acid, which led to the production of cells expressing markers for the renal lineage cells. In some reports, the induced cells formed tubule-like structures in, or were incorporated into, developing mouse kidneys $[6,26]$.

In contrast, a small number of reports have described the directed differentiation of renal lineages from human ESCs or iPSCs [6]. As a first step for the generation of renal progenitors by mimicking in vivo development, our group recently published a method for inducing the highly efficient differentiation of human iPSCs/ESCs into IM cells [27]. These human IM cells have the developmental potential to further differentiate into embryonic renal progenitors and adult renal cell types, such as glomerular podocytes and renal tubular cells. Furthermore, they can form 3-dimensional renal tubular structures in an organ culture setting. As a next step, our group is currently developing a method for the highly efficient differentiation from human iPSC/ESC-derived IM cells into kidney precursors including nephron progenitors.

Research aiming to artificially create nephron progenitors from fully differentiated adult renal cells has already been performed via cellular reprogramming. It was reported that forced expression of 6 transcription factors (SIX1, SIX2, OSR1, EYA1, HOXA11, and SNAI2) in an adult proximal tubule cell line resulted in the activation of a network of genes consistent with nephron progenitors [8]. The in vitro reprogramming of somatic cell types other than renal cells, such as fibroblasts, into renal progenitors remains to be achieved.

In summary, the generation of renal progenitors from ESCs/iPSCs via directed differentiation or from adult renal cells via cellular reprogramming would provide a po- 
tential source of embryonic renal progenitors, although the methods needed for such strategies have not yet been established. Considering the difficulty in obtaining a sufficient number of cell samples from embryos, especially human embryos, these strategies might become important for the clinical application of renal progenitors. There have so far been no reports describing the transplantation of renal progenitors generated from ESCs/ iPSCs or reprogrammed from other cell types into AKI models. Therefore, further research is needed to evaluate their therapeutic potential for kidney diseases, including AKI.

\section{Conclusion}

The development of a novel therapeutic strategy for treating AKI using renal progenitors has just started. Continued efforts to isolate and characterize renal pro- genitors from embryonic or adult kidneys, and to generate renal progenitors from ESCs/iPSCs via directed differentiation or from other cell types via cellular reprogramming, are needed. Collective and integrative research combining developmental biology, stem cell biology, and basic and clinical nephrology will therefore be required to achieve this goal.

\section{Acknowledgements}

The author would like to thank the members of the Center for iPS Cell Research and Application (CiRA), Kyoto University, for helpful discussions, and apologize to authors whose studies could not be cited owing to space limitations. This research is supported by the Japan Society for the Promotion of Science (JSPS) through its Funding Program for World-Leading Innovative R\&D on Science and Technology (FIRST Program), and by the Japan Science and Technology Agency (JST) through its Projects for Technological Development, Research Center Network for Realization of Regenerative Medicine research grant.

\section{References}

1 Chertow GM, Burdick E, Honour M, Bonventre JV, Bates DW: Acute kidney injury, mortality, length of stay, and costs in hospitalized patients. J Am Soc Nephrol 2005;16:33653370.

2 Morigi M, Benigni A: Mesenchymal stem cells and kidney repair. Nephrol Dial Transplant 2013;28:788-793

3 Evans M: Discovering pluripotency: 30 years of mouse embryonic stem cells. Nat Rev Mol Cell Biol 2011;12:680-686.

4 Thomson JA, Itskovitz-Eldor J, Shapiro SS, Waknitz MA, Swiergiel JJ, Marshall VS, Jones JM: Embryonic stem cell lines derived from human blastocysts. Science 1998;282:11451147.

5 Takahashi K, Yamanaka S: Induced pluripotent stem cells in medicine and biology. Development 2013;140:2457-2461.

-6 Osafune K: iPS cell technology-based research for the treatment of diabetic nephropathy. Semin Nephrol 2012;32:479-485.

7 Harari-Steinberg O, Pleniceanu O, Dekel B: Selecting the optimal cell for kidney regeneration: fetal, adult or reprogrammed stem cells. Organogenesis 2011;7:123-134.

-8 Hendry CE, Vanslambrouck JM, Ineson J, Suhaimi N, Takasato M, Rae F, Little MH: Direct transcriptional reprogramming of adult cells to embryonic nephron progenitors. J Am Soc Nephrol 2013;24:1424-1434.

-9 Dressler GR: Advances in early kidney specification, development and patterning. Development 2009;136:3863-3874.
10 Osafune K, Takasato M, Kispert A, Asashima M, Nishinakamura R: Identification of multipotent progenitors in the embryonic mouse kidney by a novel colony-forming assay. Development 2006;131:151-161.

11 Kobayashi A, Valerius MT, Mugford JW, Carroll TJ, Self M, Oliver G, McMahon AP: Six 2 defines and regulates a multipotent selfrenewing nephron progenitor population throughout mammalian kidney development. Cell Stem Cell 2008;3:169-181.

12 Lusis M, Li J, Ineson J, Christensen ME, Rice $\mathrm{A}$, Little $\mathrm{MH}$ : Isolation of clonogenic, longterm self renewing embryonic renal stem cells. Stem Cell Res 2010;5:23-39.

13 Humphreys BD, Valerius MT, Kobayashi A, Mugford JW, Soeung S, Duffield JS, McMahon AP, Bonventre JV: Intrinsic epithelial cells repair the kidney after injury. Cell Stem Cell 2008;2:284-291.

14 Diep CQ, Ma D, Deo RC, Holm TM, Naylor RW, Arora N, Wingert RA, Bollig F, Djordjevic G, Lichman B, Zhu H, Ikenaga T, Ono F, Englert C, Cowan CA, Hukriede NA, Handin RI, Davidson AJ: Identification of adult nephron progenitors capable of kidney regeneration in zebrafish. Nature 2011;470:95-100.

15 Harari-Steinberg O, Metsuyanim S, Omer D, Gnatek Y, Gershon R, Pri-Chen S, Ozdemir DD, Lerenthal Y, Noiman T, Ben-Hur H, Vaknin Z, Schneider DF, Aronow BJ, Goldstein RS, Hohenstein P, Dekel B: Identification of human nephron progenitors capable of generation of kidney structures and functional repair of chronic renal disease. EMBO Mol Med 2013;5:1556-1568.
16 Oliver JA, Maarouf O, Cheema FH, Martens TP, Al-Awqati Q: The renal papilla is a niche for adult kidney stem cells. J Clin Invest 2004; 114:795-804.

17 Maeshima A, Yamashita S, Nojima Y: Identification of renal progenitor-like tubular cells that participate in the regeneration processes of the kidney. J Am Soc Nephrol 2003;14: 3138-3146.

18 Kinomura M, Kitamura S, Tanabe K, Ichinose K, Hirokoshi K, Takazawa Y, Kitayama H, Nasu T, Sugiyama H, Yamasaki Y, Sugaya T, Maeshima Y, Makino H: Amelioration of cisplatin-induced acute renal injury by renal progenitor-like cells derived from the adult rat kidney. Cell Transplant 2008;17:143-158.

19 Sagrinati C, Netti GS, Mazzinghi B, Lazzeri E, Liotta F, Frosali F, Ronconi E, Meini C, Gacci M, Squecco R, Carini M, Gesualdo L, Francini F, Maggi E, Annunziato F, Lasagni L, Serio M, Romagnani S, Romagnani P: Isolation and characterization of multipotent progenitor cells from the Bowman's capsule of adult human kidneys. J Am Soc Nephrol 2006;17: 2443-2456

20 Romagnani P, Remuzzi G: Renal progenitors in non-diabetic and diabetic nephropathies. Trends Endocrinol Metab 2013;24:13-20.

-21 Rizzo P, Perico N, Gagliardini E, Novelli R, Alison MR, Remuzzi G, Benigni A: Nature and mediators of parietal epithelial cell activation in glomerulonephritides of human and rat. Am J Pathol 2013;183:1769-1778. 
22 Pippin JW, Sparks MA, Glenn ST, Buitrago S, Coffman TM, Duffield JS, Gross KW, Shankland SJ: Cells of renin lineage are progenitors of podocytes and parietal epithelial cells in experimental glomerular disease. Am J Pathol 2013;183:542-557.

-23 Hishikawa K, Marumo T, Miura S, Nakanishi A, Matsuzaki Y, Shibata K, Ichiyanagi T, Kohike H, Komori T, Takahashi I, Takase O, Imai $\mathrm{N}$, Yoshikawa $\mathrm{M}$, Inowa $\mathrm{T}$, Hayashi $\mathrm{M}$, Nakaki T, Nakauchi H, Okano H, Fujita T: Musculin/MyoR is expressed in kidney side population cells and can regulate their function. J Cell Biol 2005;169:921-928.
24 Challen GA, Bertoncello I, Deane JA, Ricardo SD, Little MH: Kidney side population reveals multilineage potential and renal functional capacity but also cellular heterogeneity. J Am Soc Nephrol 2006;17:1896-1912.

25 Dekel B, Zangi L, Shezen E, Reich-Zeliger S, Eventov-Friedman S, Katchman H, JacobHirsch J, Amariglio N, Rechavi G, Margalit R, Reisner Y: Isolation and characterization of nontubular sca-1+lin- multipotent stem/progenitor cells from adult mouse kidney. J Am Soc Nephrol 2006;17:3300-3314.
26 Kim D, Dressler GR: Nephrogenic factors promote differentiation of mouse embryonic stem cells into renal epithelia. J Am Soc Nephrol 2005;16:3527-3534.

27 Mae S, Shono A, Shiota F, Yasuno T, Kajiwara M, Gotoda-Nishimura N, Arai S, Sato-Otubo A, Toyoda T, Takahashi K, Nakayama N, Cowan CA, Aoi T, Ogawa S, McMahon AP, Yamanaka S, Osafune K: Monitoring and robust induction of nephrogenic intermediate mesoderm from human pluripotent stem cells. Nat Commun 2013;4:1367. 